\title{
Quantum-fluid-dynamics approach for strong-field processes: Application to the study of multiphoton ionization and high-order harmonic generation of He and $\mathrm{Ne}$ atoms in intense laser fields
}

\author{
Amlan K. Roy and Shih-I Chu \\ Department of Chemistry, University of Kansas, and Kansas Center for Advanced Scientific Computing, Lawrence, Kansas 66045 \\ (Received 25 July 2001; revised manuscript received 12 November 2001; published 18 March 2002)
}

\begin{abstract}
We explore the feasibility of extending the quantum-fluid dynamics (QFD) approach for quantitative investigation of nonlinear optical processes of many-electron quantum systems in intense laser fields. Through the amalgamation of the QFD and density-functional theory (DFT), a single time-dependent hydrodynamical equation of motion can be derived. This equation has the form of a generalized nonlinear Schrödinger equation (GNLSE) but includes the many-body effects through a local time-dependent exchange-correlation potential. The time-dependent generalized pseudospectral method is extended to the solution of the GNLSE in spherical coordinates, allowing nonuniform spatial discretization and efficient, accurate solution of the hydrodynamical density and wave function in space and time. The procedure is applied to the study of multiphoton ionization (MPI) and high-order harmonic generation (HHG) of $\mathrm{He}$ and $\mathrm{Ne}$ atoms in intense laser fields. Excellent agreement with other recent self-interaction-free time-dependent DFT calculations is obtained for He, while for $\mathrm{Ne}$, good agreement is achieved. Four different exchange-correlation energy functionals are used in the study with an aim to explore the roles of exchange and correlation on MPI/HHG processes in details. The method offers a conceptually appealing and computationally practical approach for nonperturbative treatment of strong-field processes of many-electron systems beyond the time-dependent Hartree-Fock level.
\end{abstract}

DOI: 10.1103/PhysRevA.65.043402 PACS number(s): 42.50.Hz, 32.80.Wr, 32.80.Qk, 71.15.Mb

\section{INTRODUCTION}

The recent advancement in the technology of highintensity and ultrashort laser pulses has led to the discovery of a number of very high order nonlinear optical phenomena in atomic, molecular, and cluster systems. Among these novel high-intensity phenomena, multiple high-order harmonic generation $(\mathrm{HHG})$ is of particular current interest. The generation of harmonics of orders in excess of 300 from rare-gas atoms has been recently demonstrated by experiments [1-3] using laser pulses shorter than $20 \mathrm{fs}$ and peak intensity more than $10^{14} \mathrm{~W} / \mathrm{cm}^{2}$. To describe such intensefield processes using $a b$ initio wave-function approach, it is necessary to solve the time-dependent Schrödinger equation of many-electron systems, which is beyond the capability of current computer technology. Development of alternative approximate approach capable of treating many-electron quantum dynamics, including electron correlation and core excitation, is thus very desirable and of considerable current interest in strong-field atomic and molecular physics.

Recently, self-interaction-free time-dependent densityfunctional theory (TDDFT) has been developed [4-6] for nonperturbative treatment of multiphoton dynamics of manyelectron quantum systems in strong fields. The main feature of TDDFT approach is a set of time-dependent Kohn-Shamlike equations that are structurally similar to the timedependent Hartree-Fock (TDHF) equations but include in principle exactly all many-body effects through a local timedependent exchange-correlation (XC) potential. Unlike the conventional DFT/TDDFT calculations using explicit XC energy functionals [7], which contain self-interaction-energy and incorrect long-range potential, these recent TDDFT approaches [4-6] are self-interaction free and the $X C$ poten- tials possess proper asymptotic long-range Coulombic behavior. These $X C$ potentials are constructed by means of the extension of the optimized-effective-potential (OEP) formalism [8] to the time domain, using either the Hartree-Fock (nonlocal) exchange energy functional [6] or the incorporation of an explicit self-interaction-correction (SIC) term [4,5]. The TDDFT/OEP-SIC approach has been recently applied successfully to the study of multiphoton ionization (MPI) and HHG processes of rare-gas atoms [4], $\mathrm{H}_{2}$ [5], and $\mathrm{N}_{2}$ [9] molecules in intense laser-pulsed fields.

In this paper, we consider an alternative approach, based on the hydrodynamic formulation of quantum mechanics. The quantum-fluid-dynamics (QFD) concept was originally proposed years ago by Madelung [10], de Broglie [11], and Bohm [12]. The QFD formulation requires solving a set of nonlinear partial-differential equations and thus is more complicated than the linear Schrödinger equation. However, the QFD has the conceptually appealing feature, namely, the electron cloud is treated as a "classical fluid" moving under the influence of classical Coulomb forces and an additional quantum potential. Recently there is also a renewed interest in extending the QFD approach as a practical tool for solving time-dependent Schrödinger equation [13-16]. This is partly related to the recognition that the amplitude and the phase of the wave function (treated explicitly in the fluid formulation as independent variables) are more slowly varying in time than the complex wave function itself, leading to computational advantages and additional physical insights of quantum dynamics or quantum trajectory [13-16]. However, $a b$ initio QFD approach has not been applied to strong-field processes even for the one-electron system involving the Coulomb potential. For many-electron systems, $a b$ initio QFD method encounters similar level of computational chal- 
lenges as the time-dependent Schrödinger equation approach.

An alternative QFD approach for many-particle systems was developed earlier by Bloch [17] within the framework of time-dependent Thomas-Fermi (TDTF) model $[18,19]$. The TDTF method can be considered as a crude version of TDDFT where the electronic system is considered as a gas of almost free electrons. More rigorous QFD formulations of DFT were treated in 1980's [20-22]. A unique feature of the combined QFD and DFT approach is that a single generalized nonlinear Schrödinger-like equation can be derived [23]. This is different from the conventional TDDFT or the recent self-interaction-free TDDFT formalisms [4-6] where individual occupied spin orbital is treated explicitly. The QFDDFT approach has been applied to the study of both static ground-state calculations (via imaginary time-evolution technique) $[24,25]$ and dynamical processes $[23,26-30]$ in cylindrical coordinates.

The motivations of this paper are threefold. First we perform a precision calculation of the nonlinear QFD-DFT equation in spherical coordinates by means of the timedependent generalized pseudospectral (TDGPS) technique $[4,5,31]$ recently developed, with an aim to assess the usefulness of QFD-DFT approach for realistic and quantitative investigation of strong-field processes. The TDGPS method allows nonuniform spatial-grid discretization and has been shown to be capable of providing high-precision timedependent wave function with the use of only a modest number of grid points. The procedure has been applied successfully to the study of HHG processes $[4,5,9,31,32]$ of atoms and molecules and Rydberg-atom high-resolution spectroscopy $[33,34]$ in external fields. Second, we extend the QFDDFT formalism to a detailed case study of the photoionization and photoemission dynamics of $\mathrm{He}$ atoms in intense laser-pulsed fields and explore the effects of "exchange" and "correlation" in details. Third, the feasibility of the formalism for more than two-electron systems is tested by applying to the case of Ne. This will be a testing ground of the formalism as more than one orbitals are involved.

The layout of the paper is as follows. In Sec. II, we present the QFD-DFT formalism and relevant equations in spherical coordinates. The numerical procedure is outlined in Sec. III. Application of the method to the study of MPI and HHG of He is given in Sec. IV. Section V presents results for the $\mathrm{Ne}$ atoms. This is followed by a few conclusions in Sec. V.

\section{THE TIME-DEPENDENT QFD-DFT FORMALISM}

The basic idea is to obtain a link between QFD and DFT for a many-electron system through the single-particle density. Here we present a brief account of the QFD-DFT formalism. A more detailed account may be found in the recent review [29]. All the electrons are regarded to be distributed over the three-dimentional (3D) space-like continuous "classical" fluid. The two basic equations in terms of the local variables, electron density $\rho(\mathbf{r}, t)$, and current density $\mathbf{j}(\mathbf{r}, t)$, can be written as (atomic units used unless otherwise mentioned): (i) the equation of continuity,

$$
\frac{\partial \rho(\mathbf{r}, t)}{\partial t}+\boldsymbol{\nabla} \cdot \mathbf{j}(\mathbf{r}, t)=0
$$

and (ii) the Euler-type equation of motion,

$$
\frac{\partial \chi(\mathbf{r}, t)}{\partial t}+\frac{1}{2}(\nabla \chi)^{2}+\frac{\delta G[\rho]}{\delta \rho}+\frac{\delta E_{\mathrm{el}-\mathrm{el}}[\rho]}{\delta \rho}+v(\mathbf{r}, t)=0,
$$

where $\mathbf{j}(\mathbf{r}, t)=\rho \boldsymbol{\nabla} \chi(\mathbf{r}, t), \chi(\mathbf{r}, t)$ being the velocity potential. $E_{\text {el-el }}$ represents the interelectronic Coulomb repulsion energy, $v(\mathbf{r}, t)$ is the external potential including electronnuclear attraction and the laser-atom interaction potential, while $G[\rho]$ is a universal density-functional comprising the kinetic and $\mathrm{XC}$ energy contributions,

$$
\begin{aligned}
G[\rho] & =T_{w}[\rho]+T_{\text {corr }}[\rho]+E_{\mathrm{XC}}[\rho], \\
& =\frac{1}{8} \int \frac{|\nabla \rho|^{2}}{\rho} d \mathbf{r}+T_{\text {corr }}[\rho]+E_{\mathrm{XC}}[\rho] .
\end{aligned}
$$

In the above equation, $T_{w}[\rho]$ signifies the Weizsäcker kinetic-energy functional [7] that is exact for one- and twoelectron Hartree-Fock systems. Therefore, the kinetic-energy correction term $T_{\text {corr }}[\rho]$ vanishes for such systems. For other systems, $T_{w}[\rho]$ alone is not enough, and the exact form of $T_{\text {corr }}[\rho]$ is unknown and must be approximated:

$$
T_{\text {exact }} \cong T_{w}[\rho]+T_{\text {corr }}[\rho] \text {. }
$$

An approximate form for $T_{\text {corr }}[\rho]$ is available for rare-gas atoms [35]. This has yielded good-quality results for electronic-structure calculations for rare-gas atoms $(\mathrm{Ne}, \mathrm{Ar}$, $\mathrm{Kr}$, and $\mathrm{Xe}$ ) [24,25]. In the present calculation for $\mathrm{Ne}$, we employ this functional form written as a sum of several Gaussians, viz.,

$$
T_{\text {corr }}=C_{k} \int f(\mathbf{r}) \rho^{5 / 3}(\mathbf{r}) d \mathbf{r} ; \quad C_{k}=(3 / 10)\left(3 \pi^{2}\right)^{2 / 3} .
$$

Here $f(\mathbf{r})$ signifies an $\mathbf{r}$-dependent term satisfying the boundary conditions $f(\mathbf{r}) \rightarrow 0$ as $r \rightarrow 0$ and $f(\mathbf{r}) \rightarrow 1$ as $r \rightarrow \infty$. The functional derivative is given by,

$$
\frac{\delta T_{\text {corr }}[\rho]}{\delta \rho}=\frac{5}{3} C_{k} \rho^{2 / 3}(\mathbf{r}) g(\mathbf{r}),
$$

where

$$
g(\mathbf{r})=\frac{3}{5} f(\mathbf{r})+\frac{2}{5} \rho^{-2 / 3}(\mathbf{r}) \int \rho\left(\mathbf{r}^{\prime}\right) \tilde{\rho}\left(\mathbf{r}^{\prime}\right)^{-1 / 3} \frac{\delta \tilde{\rho}\left(\mathbf{r}^{\prime}\right)}{\delta \rho(\mathbf{r})} d \mathbf{r}^{\prime},
$$

$\tilde{\rho}(\mathbf{r})=\rho(\mathbf{r}) f^{3 / 2}(\mathbf{r})$, and 


$$
\begin{aligned}
& f(r)=\sum_{i=1}^{n} A_{i} \exp \left[-\alpha_{i}\left(r-R_{i}\right)^{2}\right], \\
& g(r)=\sum_{i=1}^{n} A_{i} \exp \left[-\beta_{i}\left(r-R_{i}\right)^{2}\right] .
\end{aligned}
$$

The values of $A_{i}, R_{i}, \alpha_{i}$, and $\beta_{i}$ for $\mathrm{Ne}, \mathrm{Ar}, \mathrm{Kr}$, and $\mathrm{Xe}$ are given in Ref. [25].

Equations (2.1) and (2.2) can be combined into a single equation by defining the complex-valued TD hydrodynamical wave function for the entire time-evolving system as (in polar form)

$$
\Psi(\mathbf{r}, t)=\rho(\mathbf{r}, t)^{1 / 2} e^{i \chi(\mathbf{r}, t)},
$$

and eliminating $\chi(\mathbf{r}, t)$ from them. The result is a generalized nonlinear Schrödinger equation:

$$
\left[-\frac{1}{2} \nabla^{2}+v_{e f f}([\rho] ; \mathbf{r}, t)\right] \Psi(\mathbf{r}, t)=i \frac{\partial \Psi(\mathbf{r}, t)}{\partial t},
$$

where $\quad \rho(\mathbf{r}, t)=|\Psi(\mathbf{r}, t)|^{2}$. The effective potential $v_{\text {eff }}([\rho] ; \mathbf{r}, t)$ contains both classical and quantum potentials,

$$
v_{\mathrm{eff}}([\rho] ; \mathbf{r}, t)=\frac{\delta E_{\mathrm{el}-\mathrm{el}}}{\delta \rho}+\frac{\delta \mathrm{E}_{\mathrm{nu}-\mathrm{el}}}{\delta \rho}+\frac{\delta E_{\mathrm{XC}}}{\delta \rho}+\frac{\delta T_{\mathrm{corr}}}{\delta \rho}+\frac{\delta E_{\mathrm{ext}}}{\delta \rho},
$$

where the first two potentials have their usual significance,

$$
\frac{\delta E_{\mathrm{el}-\mathrm{el}}[\rho]}{\delta \rho}=\int \frac{\rho\left(\mathbf{r}^{\prime}, t\right)}{\left|\mathbf{r}-\mathbf{r}^{\prime}\right|} d \mathbf{r}^{\prime} ; \quad \frac{\delta E_{\text {nu-el }[}[\rho]}{\delta \rho}=-\frac{Z}{r} .
$$

$E_{\mathrm{XC}}$ is the XC energy-density functional, and $E_{\text {ext }}$ is the interaction energy of the electron with the external electric field. Various XC energy functionals within the local-density approximation (LDA) or generalized gradient approximation (GGA) are available in the literature [7]. We have employed two forms for the exchange potentials for He, viz., the exact exchange-energy expression,

$$
\frac{\delta E_{X}[\rho]}{\delta \rho}=-\frac{1}{2} \int \frac{\rho\left(\mathbf{r}^{\prime}, t\right)}{\left|\mathbf{r}-\mathbf{r}^{\prime}\right|} d \mathbf{r}^{\prime},
$$

and the local-density approximation (LDA),

$$
\frac{\delta E_{X}^{\mathrm{LDA}}[\rho]}{\delta \rho}=-\frac{4}{3} C_{X} \rho^{1 / 3}, \quad C_{X}=\frac{3}{4}\left(\frac{3}{\pi}\right)^{1 / 3} .
$$

For Ne, a local-exchange potential [36] has been employed,

$$
E_{X}=E_{X}^{\mathrm{LDA}}-\beta \int \frac{\rho^{1 / 3}}{\left(1+\frac{r^{2} \rho^{2 / 3}}{\alpha_{X}}\right)} \rho d \mathbf{r},
$$

where

$$
E_{X}^{\mathrm{LDA}}=-\mathrm{C}_{\mathrm{X}} \int \rho^{4 / 3} d \mathbf{r}
$$

and

$$
\frac{\delta E_{X}}{\delta \rho}=\frac{\delta E_{X}^{\mathrm{LDA}}}{\delta \rho}-\beta\left[\frac{\frac{4}{3} \rho^{1 / 3}+\frac{2}{3} \frac{r^{2} \rho}{\alpha_{X}}}{\left(1+\frac{r^{2} \rho^{2 / 3}}{\alpha_{X}}\right)^{2}}\right] .
$$

Here $C_{X}=(3 / 4)(3 / \pi)^{1 / 3}, \beta=C_{X}$ and $\alpha_{X}=0.02440$. This exchange functional [36] shows correct asymptotic behavior. It is also local and gradient-free; hence requires less computational effort than other GGA functionals. This functional has been found to be capable of yielding good-quality results for atomic systems [25]. For example, the exchange energies (in a.u.) calculated from Eq. (2.14) are 1.026, 12.14, 30.15, 93.94, and 179.2 for $\mathrm{He}, \mathrm{Ne}, \mathrm{Ar}, \mathrm{Kr}$, and $\mathrm{Xe}$, respectively, while the corresponding $\mathrm{HF}$ values are 1.026, 12.11, 30.19, 93.89, and 179.2, using the HF densities [36].

To include correlation effects, we use a simple, parametrized local Wigner-type functional [37], which yielded quite good results for electron structure calculations of many-electron systems, (see e.g., Refs. [38,39]). Other justifications for using this form of correlation functional can be found in Ref. [27]. The Wigner correlation potential is given by

$$
\frac{\delta E_{C}}{\delta \rho}=-\frac{a+c \rho^{-1 / 3}}{\left(a+b \rho^{-1 / 3}\right)^{2}},
$$

where $a=9.810, b=21.437$, and $c=28.582667$. Assuming the linearly polarized laser field is along the $z$ axis, the interaction potential can be written as

$$
\frac{\delta E_{\mathrm{ext}}}{\delta \rho}=-\boldsymbol{\mu}(\mathbf{r}, t) \cdot \boldsymbol{E}(t)=-z E_{0} f(t) \sin (\omega t) .
$$

Since the system has the axial symmetry, the spatial dimensionality reduces to two. In the above equation, $\boldsymbol{\mu}(\mathbf{r}, t)$ is the TD induced dipole moment, $\mathrm{E}_{0}$ is the electric-field amplitude, $f(t)$ is the laser shape pulse function, and $\omega$ is the laser frequency.

Note that if Eqs. (2.1) and (2.2) are written in imaginary time $\tau(\tau=-i t, t$ being the real time $)$ and $\chi(\mathbf{r}, t)$ is eliminated, one can obtain a diffusion-type equation, viz.,

$$
\left[-\frac{1}{2} \nabla^{2}+v_{\mathrm{eff}}([\rho] ; \mathbf{r}, t)\right] R(\mathbf{r}, t)=-\frac{\partial R(\mathbf{r}, t)}{\partial t} .
$$

The diffusion function no longer resembles the hydrodynamical function and accordingly has been replaced by $R(\mathbf{r}, t)$. This equation has been used for accurate calculations of the static ground-state properties of many-electron systems $[24,25]$.

Equation (2.9) is highly nonlinear; nonlinearity arising mainly through the nonintegral powers of $\rho$ and the integrals in $v_{e f f}([\rho] ; \mathbf{r}, t)$. This equation goes much beyond the TDHF or the linear-response approximation, since it calculates 
$\rho(\mathbf{r}, t)$ to all orders of changes, which is then employed to obtain the various TD quantities of interest. It may be noted that the Laplacian arises from the Weizsäcker term and not from the fictitious "noninteracting" kinetic energy as in the Kohn-Sham theory. Numerical solution of Eq. (9) yields the TD effective potential and a single "hypothetical" TD orbital for the entire interacting system.

\section{NUMERICAL PROCEDURE}

It is known that the equal-spacing spatial-discretization methods require a very large number of grid points to get the converged results. The generalized pseudospectral (GPS) method [40,41] alleviates such problems and allows nonuniform and optimal spatial-grid discretization: denser mesh at shorter distances and sparser mesh at longer range. This is important for the $\mathrm{HHG}$, because it is the probability density near the origin that determines the dipole acceleration. In this section, we extend the numerical scheme of the timedependent GPS method [31] to the solution of Eq. (2.9). Denoting the nonlinear operator in square bracket by $\hat{L}$, Eq. (2.9) can be recast in to the following form:

$$
\frac{\partial \Psi(\mathbf{r}, t)}{\partial t}=-i \hat{L} \Psi(\mathbf{r}, t)=-i\left[\hat{H}_{0}+\hat{V}\right] \Psi(\mathbf{r}, t),
$$

i.e., $\partial / \partial t=-i \hat{L}$. Here $\hat{\mathrm{H}}_{0}$ denotes the "unperturbed" operator and includes potentials arising from the Coulomb repulsion, $E_{\mathrm{XC}}$, nuclear-electron attraction and the nonclassical kinetic-energy correction term $T_{\text {corr }}$ at the zero time. The operator $\hat{V}$ consists of two terms: the classical external interaction potential $v_{\text {ext }}(\mathbf{r}, t)$ as well as the "residual" effective potential $v_{\text {res }}(\mathbf{r}, t)$,

$$
\begin{gathered}
\hat{V}(\mathbf{r}, t)=v_{\mathrm{ext}}(\mathbf{r}, t)+v_{\mathrm{res}}(\mathbf{r}, t), \\
v_{\mathrm{res}}(\mathbf{r}, t)=v_{\mathrm{eff}}(\mathbf{r}, t)-v_{\mathrm{eff}}(\mathbf{r}, 0) .
\end{gathered}
$$

The time propagation is implemented by the use of secondorder split-operator scheme in the energy representation in spherical coordinates $[4,31]$,

$$
\begin{aligned}
\Psi(\mathbf{r}, t+\Delta t) \simeq & e^{\left(-i \hat{H}_{0} \Delta t / 2\right)} \exp [-i \hat{V}(r, \theta, t+\Delta t / 2) \Delta t] \\
& \times e^{\left(-i \hat{H}_{0} \Delta t / 2\right)} \Psi(\mathbf{r}, t)+O\left(\Delta t^{3}\right)
\end{aligned}
$$

This is different from other commonly used split-operator schemes in the literature $[42,43]$ where $\hat{\mathrm{H}}_{0}$ is generally taken to be the radial kinetic-energy operator and $\hat{V}$ the remaining Hamiltonian depending on the spatial coordinates only. The advantages of using the energy representation has been discussed elsewhere [4,5,31].

Now the hydrodynamical wave function $\Psi(\mathbf{r}, t)$ is expanded in the Legendre polynomial basis,

$$
\Psi\left(r_{i}, \theta_{j}, t\right)=\sum_{l} \quad g_{l}\left(r_{i}, t\right) P_{l}\left(\cos \theta_{j}\right),
$$

and the GPS method $[40,41]$ is used to discretize the radial coordinate $r$. Detailed TDGPS procedures can be found in Refs. [4,31]. To prevent back reflection from the boundary, an absorber of the following form has been used in the present calculation [31],

$$
\cos ^{0.25}\left[\frac{\pi}{2}\left(r-r_{0}\right) /\left(r_{\max }-r_{0}\right)\right], \quad r \geqslant r_{0},
$$

where $r_{\max }$ is typically 150-200 a.u., while that of $r_{0}$ is 100-150 a.u. Absorber position and the grid size have been chosen carefully such that only those parts that are traveling away from the nucleus and will not return are absorbed.

Once the hydrodynamical function is obtained, the TD density can be calculated that yields all other density-based "observables" of the process. The rich dynamics of the interaction process is revealed in the TD density plots. Apart from the TD density, the following quantities have been calculated.

(1) The normalized total electronic charge $N(t)$ at a particular instant $t$ is given by,

$$
N(t)=\int \rho(\mathbf{r}, t) d \mathbf{r} .
$$

This gives the information about the ionization probability.

(2) The excitation and ionization probability, $P_{e i}(t)$, is given by

$$
P_{e i}(t)=1-P_{g s}(t),
$$

where $P_{g s}(t)=\langle\Psi(0) \mid \Psi(t)\rangle^{2}$ is the TD ground-state population of the $\mathrm{He} / \mathrm{Ne}$ atom and is normalized to unity at $t$ $=0$.

(3) Another important "observable" is the radiated harmonic spectra emitted due to the strong perturbation. The induced dipole moment can be expressed in the length and acceleration form, respectively, as follows:

$$
d(t)=\int \rho(\mathbf{r}, t) z d \mathbf{r}
$$

and

$$
d_{A}(t)=\int \rho(\mathbf{r}, t) \frac{d^{2} z}{d t^{2}} d \mathbf{r} .
$$

The corresponding HHG power spectrum can be calculated from the modulus square of the Fourier transformation of the respective TD dipole moments in the two gauges

$$
P(\omega)=\left|\frac{1}{t_{f}-t_{i}} \int_{t_{i}}^{t_{f}} d(t) e^{-i \omega t} d t\right|^{2}
$$

and

$$
P_{A}(\omega)=\left|\frac{1}{t_{f}-t_{i}} \frac{1}{\omega^{2}} \int_{t_{i}}^{t_{f}} d_{A}(t) e^{-i \omega t} d t\right|^{2}
$$




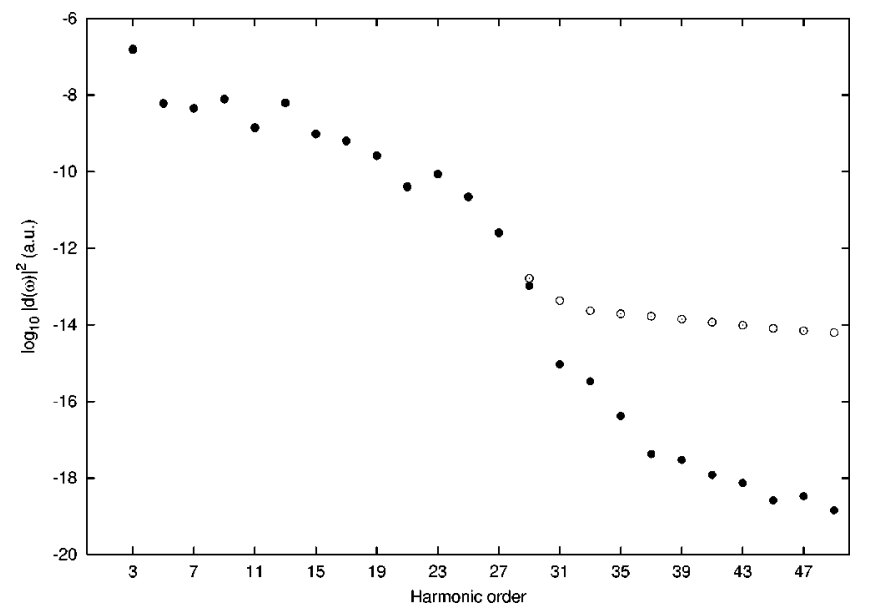

FIG. 1. Comparison of HHG spectra of He in length (open circles) and acceleration (filled circles) forms calculated from the TD QFD-DFT method. The laser intensity is $3 \times 10^{14} \mathrm{~W} / \mathrm{cm}^{2}$, wavelength is $527 \mathrm{~nm}$, and the field has a $\sin ^{2}$ pulse shape with 60 o.c. in duration.

The HHG power spectra obtained from these two expressions should be identical if the TD wave function is fully converged.

\section{MPI AND HHG OF HE ATOMS IN INTENSE LASER FIELDS}

In this section, we extend the QFD-DFT formalism to a case study of the nonlinear response of $\mathrm{He}$ atoms in intense laser fields, with particular attention to the investigation of the effects of the exchange $(X)$ and the correlation $(C)$ effects on the MPI and HHG processes. The key equation is the generalized nonlinear Schrödinger equation, Eq. (2.9), which is solved by the TDGPS method. The initial wave function at $t=0$ is obtained by the GPS discretization and solution of the corresponding time-dependent Kohn-Sham (TDKS) equation in imaginary time. Here it may be mentioned that the earlier TDKS approaches for the laser-atom interaction studies have usually employed the ground states at $t=0 \mathrm{ob}-$ tained from the solution of the corresponding timeindependent equations. In the absence of the laser fields, the unperturbed energy of the ground state is preserved to at least 10-12 digits of accuracy and the norm of the wave function maintains unity during the whole time propagation.

\section{A. Test of numerical convergence of QFD-DFT calculations}

Figure 1 shows a comparison of the HHG power spectra obtained from the length and acceleration forms, $d(t)$ and $d_{A}(t)$, respectively, for the case of linearly polarized laserpulsed fields with wavelength $527 \mathrm{~nm}(\omega=0.08646$ a.u. $)$, peak intensity $3 \times 10^{14} \mathrm{~W} / \mathrm{cm}^{2} \quad\left(E_{0}=0.09246\right.$ a.u. $), \sin ^{2}$ pulse shape and 60 optical cycles (o.c.) in pulse length. As shown, excellent agreement is achieved from the lowest harmonic, through the plateau, all the way to the cutoff, indicating the time-dependent wave function is converged. The largest disagreement (the third harmonic) is only about $1.92 \%$. The deviation of the length form spectrum from that of the acceleration form beyond the cutoff is well understood $[43,44]$. It is generally believed that the extra harmonics beyond the cutoff in the length gauge is spurious and the acceleration form is the more accurate representation. Accordingly, we will present only the acceleration HHG power spectra henceforth. In this calculation, 300 grid points are used for the Legendre pseudospectral discretization of the radial coordinate $r$ (using a nonlinear algebraic mapping $[4,31]$ to map $r=\left[0, r_{\max }\right]$ to $\left.x=[-1,1]\right)$ and 30 partial waves are used to achieve the convergence. Typically $r_{\max }$ is about 150-200 a.u., and an absorber is placed at around $r=100$ a.u. Higher accuracy can be achieved by increasing the number of grid points. We note that our HHG results are generally in excellent agreement with the recent calculation using the TDDFT/OEP-SIC method [4]. This provides additional check on the accuracy and usefulness of the present QFD-DFT approach.

\section{B. Exchange $(X)$ and correlation $(C)$ energy functionals}

In conventional steady-state DFT calculations, explicit $\mathrm{XC}$ energy functionals have been often used, including the LDA and the more refined GGA such as the Becke $(B)$ 's exchange energy functional [45] and Lee-Yang-Parr (LYP)'s [46] correlation energy functional, etc. Since exact timedependent XC energy functionals are currently not available, the common procedure in TDDFT calculations is to adopt the adiabatic approximation. Within such a framework, the XC potential $v_{\mathrm{XC}}(\mathbf{r}, t)$ can be written as the functional derivative of the XC energy $E_{\mathrm{XC}}$ with respect to the density:

$$
v_{\mathrm{XC}}(\mathbf{r}, t)=\left.\frac{\delta E_{\mathrm{XC}}[\rho]}{\delta \rho}\right|_{\rho=\rho(\mathbf{r}, t)} .
$$

It is well known that DFT/TDDFT calculations, using either LDA or GGA explicit energy functionals, contain selfinteraction energy terms and the corresponding $\mathrm{XC}$ potentials decay too fast and do not possess the correct asymptotic long-range $-1 / r$ Coulombic behavior [7]. Thus the excitedstate energies, and the ionization potentials obtained from the highest-occupied spin orbitals are not very accurate. Typically the ionization potentials are about 30 to $50 \%$ too low compared to the experimental values. To overcome such difficulties, it is essential to remove the self-interaction energy. The recent developments of the self-interaction-free DFT $[8,47]$ and TDDFT [4-6] are along this direction.

To study the effects of exchange and correlation on the intense field processes, we shall therefore consider the following four different XC energy functionals, in the order of decreasing accuracy: (i) exact Hartree-Fock (HF) exchange $(X)+$ Wigner correlation $(C)$, denoted by "exact $X+C$," (ii) exact $X$ only, (iii) LDA exchange + Wigner correlation, denoted by "LDA- $X C$," and (iv) LDA- $X$ only. The Wigner correlation energy functional is used here because of its simplicity and its reliability for atomic systems. It has been demonstrated that the Wigner correlation can provide rather accurate results for the atomic systems including two-electron atoms $[24-27,38,39]$. For example, the ground-state total energies obtained by the above four different energy func- 
tionals are, respectively, $-2.9031,-2.8609,-2.7657$, and -2.7235 a.u. All these should be compared with the BLYP value of -2.905 a.u. [47] and the experimental value of -2.9037 a.u. [48]. As shown, our (exact $X+C$ ) energy is in very good agreement with the experimental value. In the following, we study in details the effects of different XC energy functionals on the MPI/HHG processes.

\section{Effects of exchange and correlation on multiphoton ionization of $\mathrm{He}$}

In this subsection, we consider the effects of exchange and correlation on the MPI processes of He in three different laser intensities: (a) $3 \times 10^{14} \mathrm{~W} / \mathrm{cm}^{2}$, (b) $6 \times 10^{14} \mathrm{~W} / \mathrm{cm}^{2}$, and (c) $1 \times 10^{15} \mathrm{~W} / \mathrm{cm}^{2}$. The laser parameters used are wavelength $527 \mathrm{~nm}, \sin ^{2}$ pulse shape with 60 o.c. pulse length. The comparison of the time-dependent electron population, $N(t)$, for the four different XC energy functionals used [(i)-(iv) mentioned above], are shown in Figs. 2(a)2(c), for the respective laser intensities indicated above. Note that the ionization probability can be calculated by $P_{\text {ion }}(t)$ $=1-N(t)$ and the ionization rate from the slope of the decaying curves shown in Figs. 2(a)-2(c). Several general behaviors of the effects of $X$ and $C$ are noticed. First, we see that there is a substantial difference in $N(t)$ among various $\mathrm{XC}$ energy functionals used for a given laser intensity. However, (i),(ii) and (iii),(iv) tend to form two widely separated groups. The first group, (i) and (ii), has the exact exchange and correct long-range potential but they differ in the correlation (with or without Wigner correlation). The second group, (iii) and (iv), belongs to the LDA-level calculations (with or without the Wigner correlation) and does not have the correct exchange functional and proper long-range potential. Clearly the effect of exchange on MPI is much larger than that of the correlation. Thus it is essential to have the correct exchange and long-range potential in the QFD-DFT calculations. Second, as the laser intensity increases from Figs. 2(a)-2(c), we notice that the discrepancy within each group [(i),(ii) or (iii),(iv)] also increases. This indicates that effect of correlation cannot be ignored. In fact, it is more pronounced in higher laser intensities. Third, it is instructive to note that the order of ionization probability is $P(\mathrm{iv})$ $>P($ iii $)>P($ ii $)>P($ i) for all the three laser intensities, where the number in the parenthesis indicates the type of XC energy functional used. This order is consistent with the order of decreasing binding energy of the electrons. Thus the LDA electron (with $X$ only), case (iv), has the smallest binding energy and the largest ionization probability, while the electron described by exact exchange + Wigner correlation [case (i)] has the largest binding energy and smallest ionization probability, etc. That the binding energy, which is determined by the $\mathrm{XC}$ energy functional used, is the major factor accounting for the observed ionization-probability behavior in He has been also reported recently in other study using the TDDFT/OEP-SIC formalism [4]. The situation can be different for more complex atoms, where the orbital orientation can also play an important role in the MPI of atoms [4].
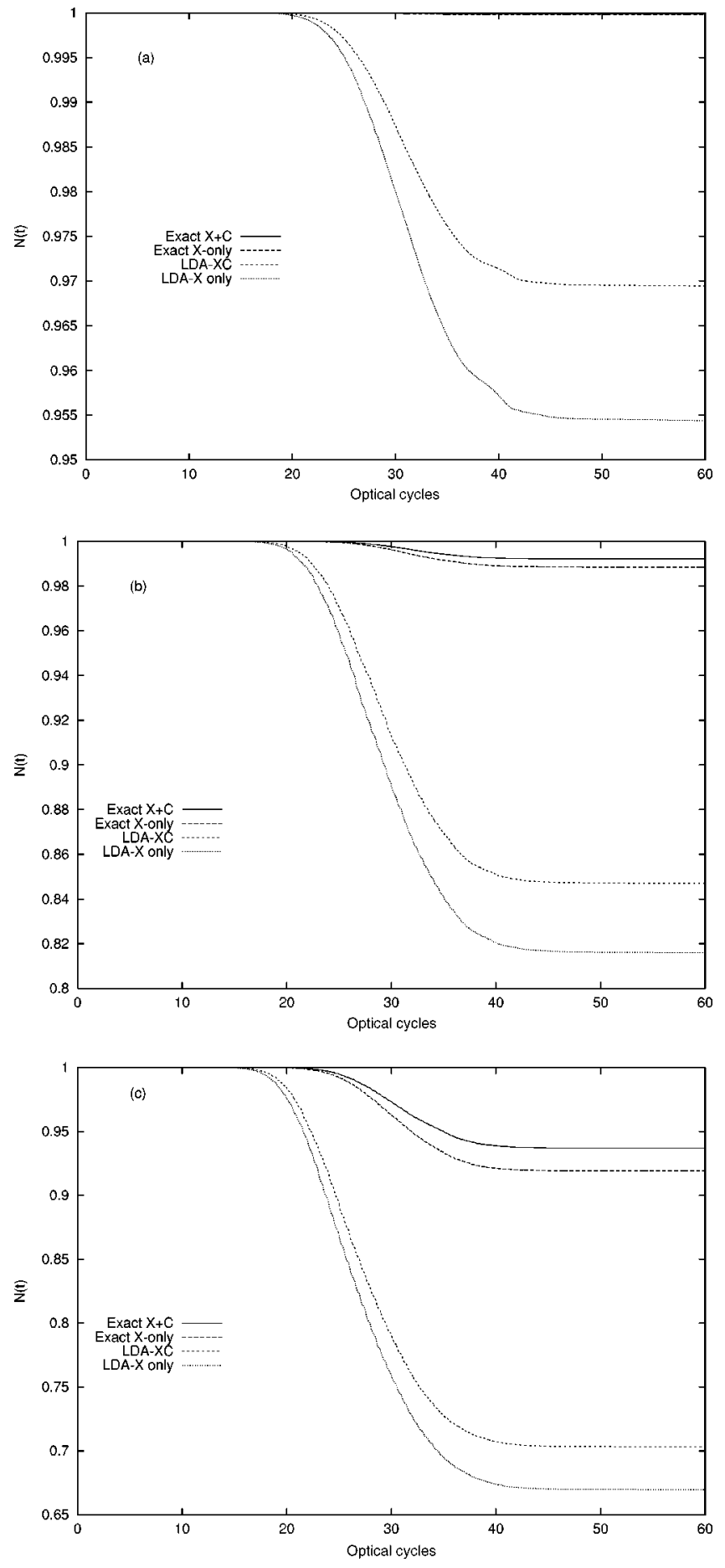

FIG. 2. Variation of the total electronic charge, $N(t)$, with time for the four different cases (i)-(iv) of XC energy functionals used (see text). (a)-(c) correspond to the laser intensities of 3 $\times 10^{14} \mathrm{~W} / \mathrm{cm}^{2}, 6 \times 10^{14} \mathrm{~W} / \mathrm{cm}^{2}$, and $1 \times 10^{15} \mathrm{~W} / \mathrm{cm}^{2}$, respectively, at the wavelength $527 \mathrm{~nm}$. $\sin ^{2}$ pulse shape is used and the pulse duration is 60 o.c.

\section{Effects of exchange and correlation on HHG of $\mathrm{He}$}

Figures 3(a)-3(c) show the comparison of the HHG power spectra of $\mathrm{He}$ for the four different energy functionals [(i)-(iv)], corresponding to the same three respective laser intensities in Figs. 2(a)-2(c). Similar to the ionization case, 

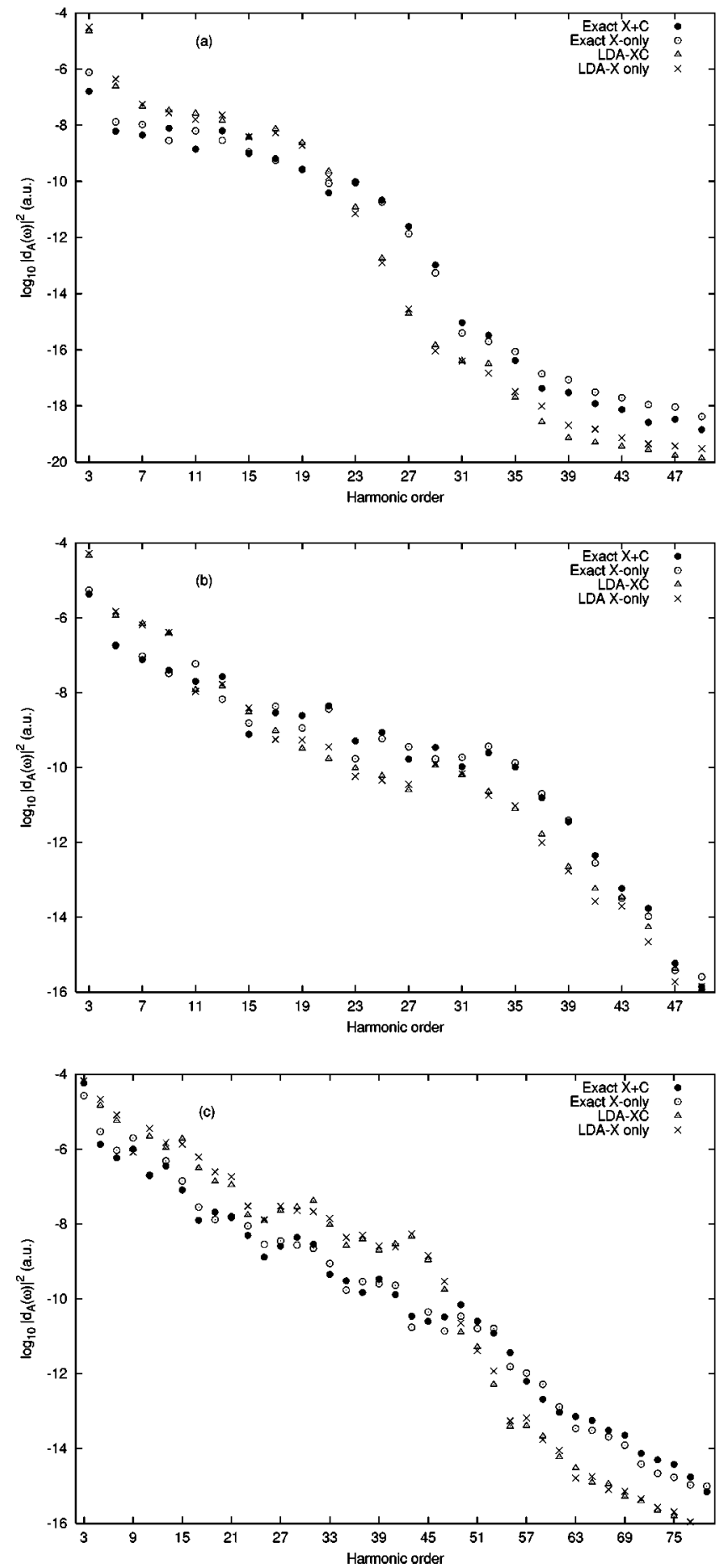

FIG. 3. Comparison of the HHG spectra of $\mathrm{He}$ atom for the four different cases (i)-(iv) of XC energy functionals used (see text). (a) - (c) correspond to the laser intensities of $3 \times 10^{14} \mathrm{~W} / \mathrm{cm}^{2}$, $6 \times 10^{14} \mathrm{~W} / \mathrm{cm}^{2}$, and $1 \times 10^{15} \mathrm{~W} / \mathrm{cm}^{2}$, respectively. The laser wavelength, pulse shape, and pulse length are the same as those in Fig. 2.

two different groups [(i),(ii) and (iii),(iv)] can be clearly distinguished and the effect of the exchange is considerably larger than that of the correlation. Typically, the effect of correlation can cause a difference of harmonic intensity by a factor of 2-3 or more while the exchange can cause $2-3$ or more orders of magnitude difference in harmonic intensity.

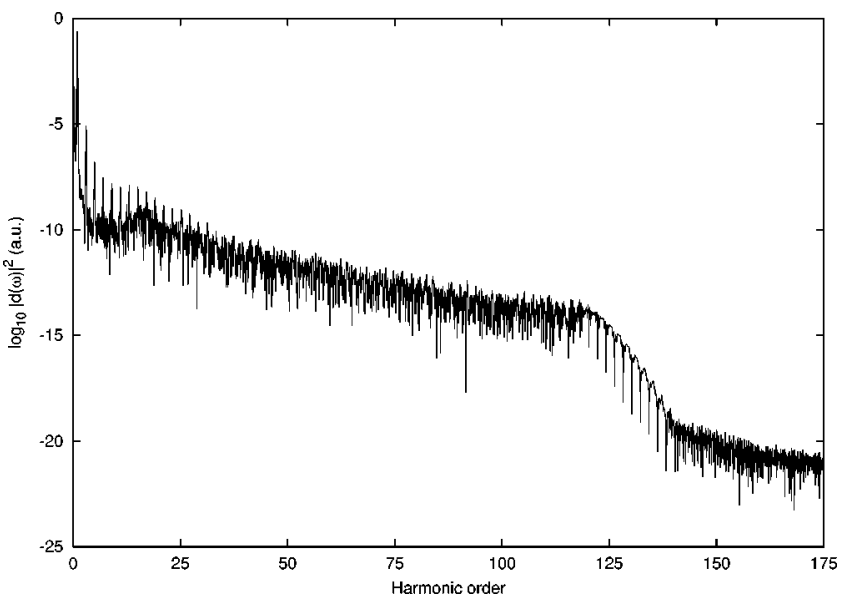

FIG. 4. Calculated HHG power spectra of He for a laser intensity of $3 \times 10^{15} \mathrm{~W} / \mathrm{cm}^{2}$ and wavelength $527 \mathrm{~nm}$. The laser field has a $\sin ^{2}$ pulse shape with 60 o.c. in duration. Exact $X+$ Wigner correlation energy functionals are used.

The relative order of HHG intensities and cutoff positions follow roughly the following pattern: the LDA calculations tend to have larger HHG intensities and earlier cutoff than those of calculations with exact exchange. Such differences may be attributed to the typical behavior of looser-bound LDA electrons versus the tighter-bound electrons; namely, an atom with smaller binding energy tends to have larger HHG intensities and shorter cutoff [4]. Due to the rather large discrepancy in using different XC energy functionals, it is clear that the effects of exchange and correlation, particularly the exchange, are both significant. To achieve quantitative study of HHG processes of many-electron systems, it is thus essential to adopt either exact exchange energy functional such as the one used here or the self-interaction-free TDDFT [4-6], both give rise to the correct asymptotic long-range potential.

\section{E. Intensity-dependent HHG power spectrum and hydrodynamical radial density distribution}

Figure 4 shows the HHG power spectrum at a stronger field with laser intensity $3.5 \times 10^{15} \mathrm{~W} / \mathrm{cm}^{2}$, wavelength 527 $\mathrm{nm}, \sin ^{2}$ pulse shape, and 30 o.c. in pulse duration. The cutoff harmonic now extends to well beyond 100. Further insight regarding the He-laser interaction is revealed in the time-dependent hydrodynamical radial density (spherically averaged) plots, particularly for the stronger-field cases. Figures 5(a) and 5(b) show the time-dependent radial densities of $\mathrm{He}$ at the two largest laser-intensity cases considered: (a) $1 \times 10^{15} \mathrm{~W} / \mathrm{cm}^{2}$, and (b) $3.5 \times 10^{15} \mathrm{~W} / \mathrm{cm}^{2}$, respectively. For case (a), we see the radial electron density is monotonically decreasing with increasing time and a small fraction of electron density leaks to larger distances. For the strongerfield case (b), the pattern is more dramatic, particularly near the peak-intensity regime (around 15 o.c., where more complicated spatial pattern is developed.

\section{MPI AND HHG OF NE ATOMS: A TEST OF TD QFD- DFT APPROACH FOR MANY-ELECTRON SYSTEMS}

In this section, we extend the TD QFD-DFT approach to the study of MPI and HHG of Ne atoms. The major differ- 

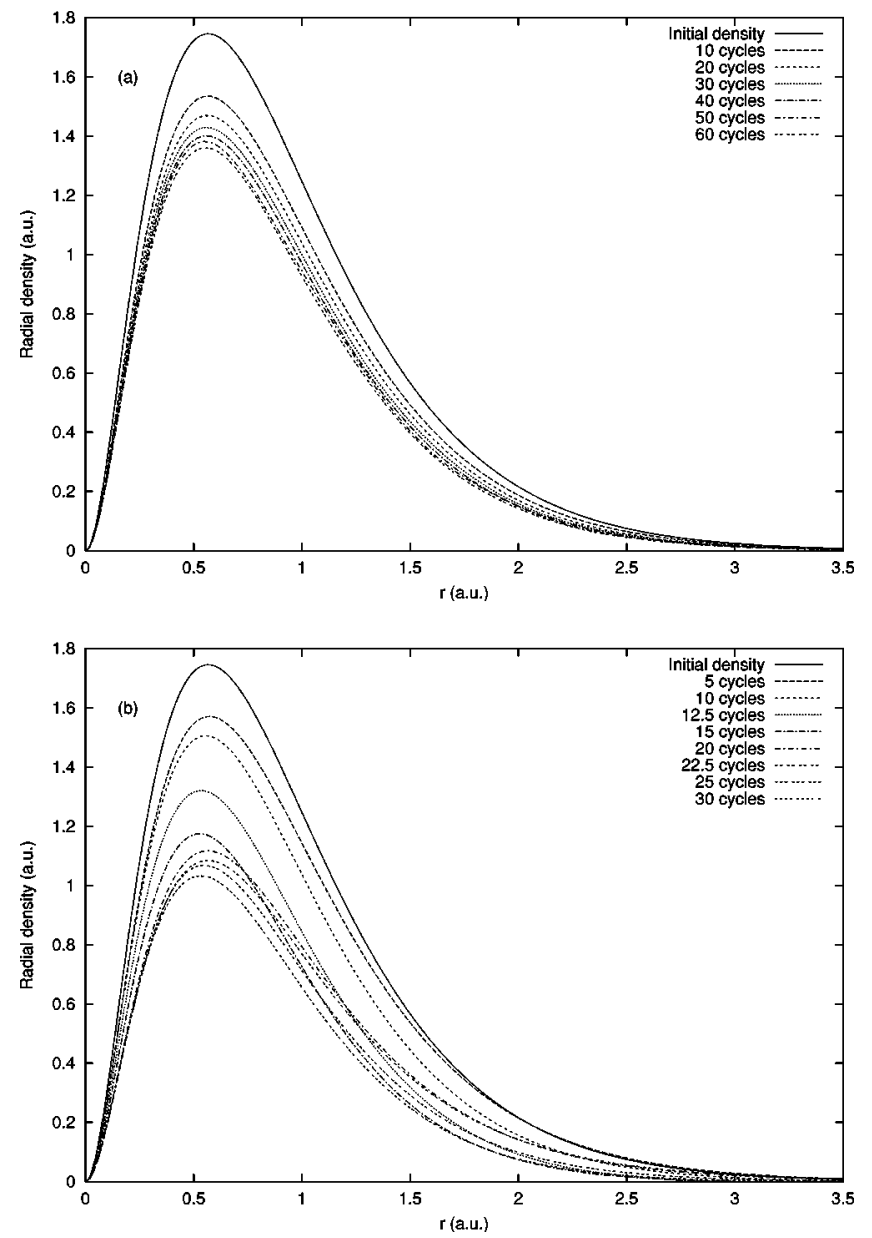

FIG. 5. Radial density plots of $\mathrm{He}$ for laser intensities (a) 1 $\times 10^{15} \mathrm{~W} / \mathrm{cm}^{2}$ and (b) $3 \times 10^{15} \mathrm{~W} / \mathrm{cm}^{2}$, at wavelength $527 \mathrm{~nm}$, $\sin ^{2}$ pulse shape and pulse duration of 60 and 30 o.c., respectively.

ence in this case from that of He is that, the modified kineticenergy term, $T_{\text {corr }}$, in Eq. (2.3), is nonzero. To see the significance of including the $T_{\text {corr }}$ term, we first present the field-free ground-state results. The total electronic energy calculated from the diffusion Eq. (2.19), using the nonclassical kinetic-energy term $T_{\text {corr }}$ in Eq. (2.4), and the exchange and correlation potentials given by Eqs. (2.16) and (2.17), respectively, yields $E=-128.9103$ a.u. Similar calculation but including the LDA-exchange energy functional in Eq. (2.15) and the same correlation functional in Eq. (2.17), gives rise to $E=-127.4513$ a.u. All of these should be compared with the best theoretical literature result of -128.939 a.u. [49]. Figure 6 gives a plot of $\delta T_{\text {corr }} / \delta \rho$ versus $\mathrm{r}$ for Ne. This apart from the total energy values clearly demonstrates that the nonclassical kinetic-energy term $T_{\text {corr }}$, in addition to $T_{w}$, is absolutely necessary to include for more than twoelectron systems to produce physically meaningful results. Moreover, the form of $T_{\text {corr }}$ given by Eqs. (2.4)-(2.7) appears to be capable of providing a good description for the electronic-structure calculations of $\mathrm{Ne}$ and other rare-gas atoms [25].

Figures 7(a) and 7(b) present the profile of the timedependent norm $N(t)$ (normalized to unity) for the two laser intensities, $5 \times 10^{13}$ and $1 \times 10^{14} \mathrm{~W} / \mathrm{cm}^{2}$, respectively, for

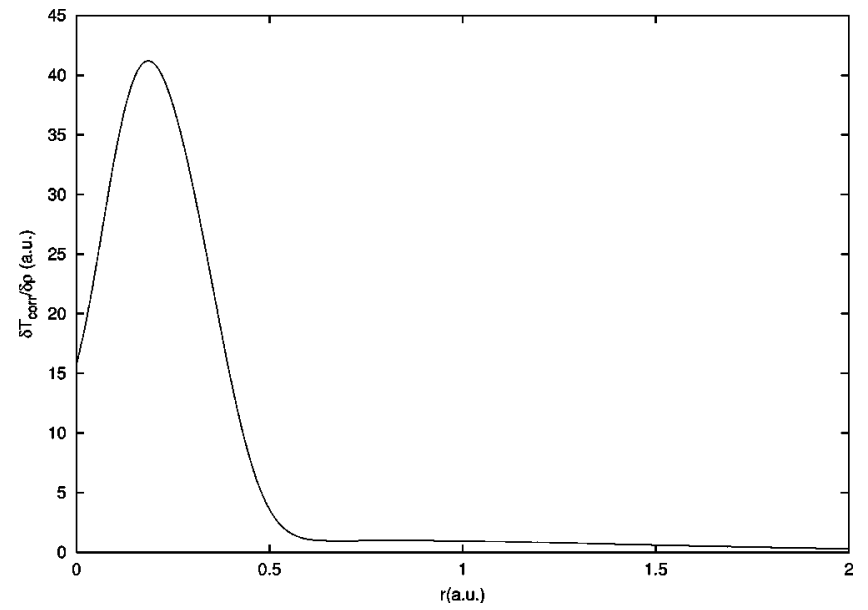

FIG. 6. Plot of $\delta T_{\text {corr }} / \delta \rho$ vs $r$, in a.u., for Ne.

the following laser parameters: $\lambda=1064 \mathrm{~nm}, \sin ^{2}$ pulse with 60 in duration. Each plot contains the comparison of the present TD QFD-DFT results [with XC potentials given by Eqs. (2.16) and (2.17)] with the individual orbital ( $2 s$ and
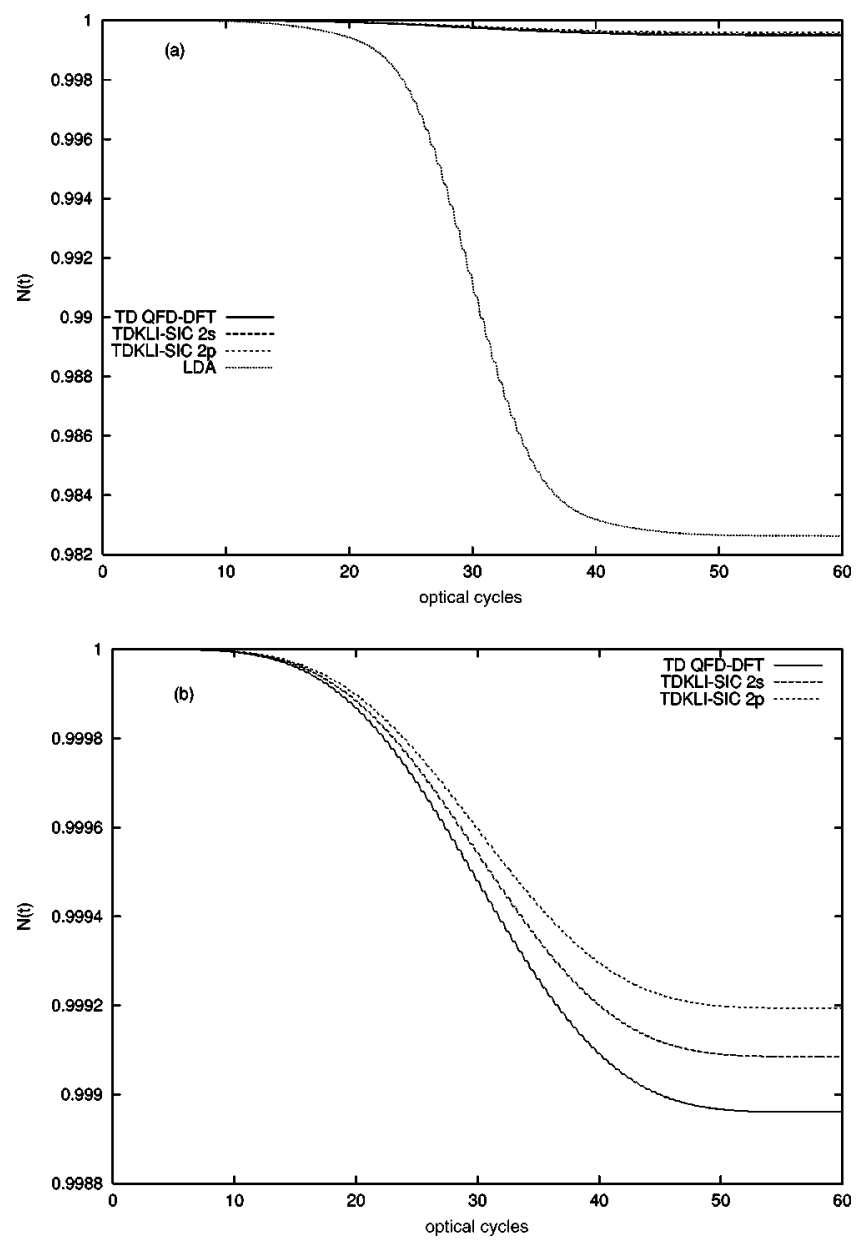

FIG. 7. Variation of the norm, $N(t)$, with time for Ne. (a) and (b) correspond to the laser intensities of $5 \times 10^{13} \mathrm{~W} / \mathrm{cm}^{2}$ and 1 $\times 10^{14} \mathrm{~W} / \mathrm{cm}^{2}$, respectively, at the wavelength $1064 \mathrm{~nm} . \sin ^{2}$ pulse shape is used and the pulse duration is 60 o.c. See text for explanation. 

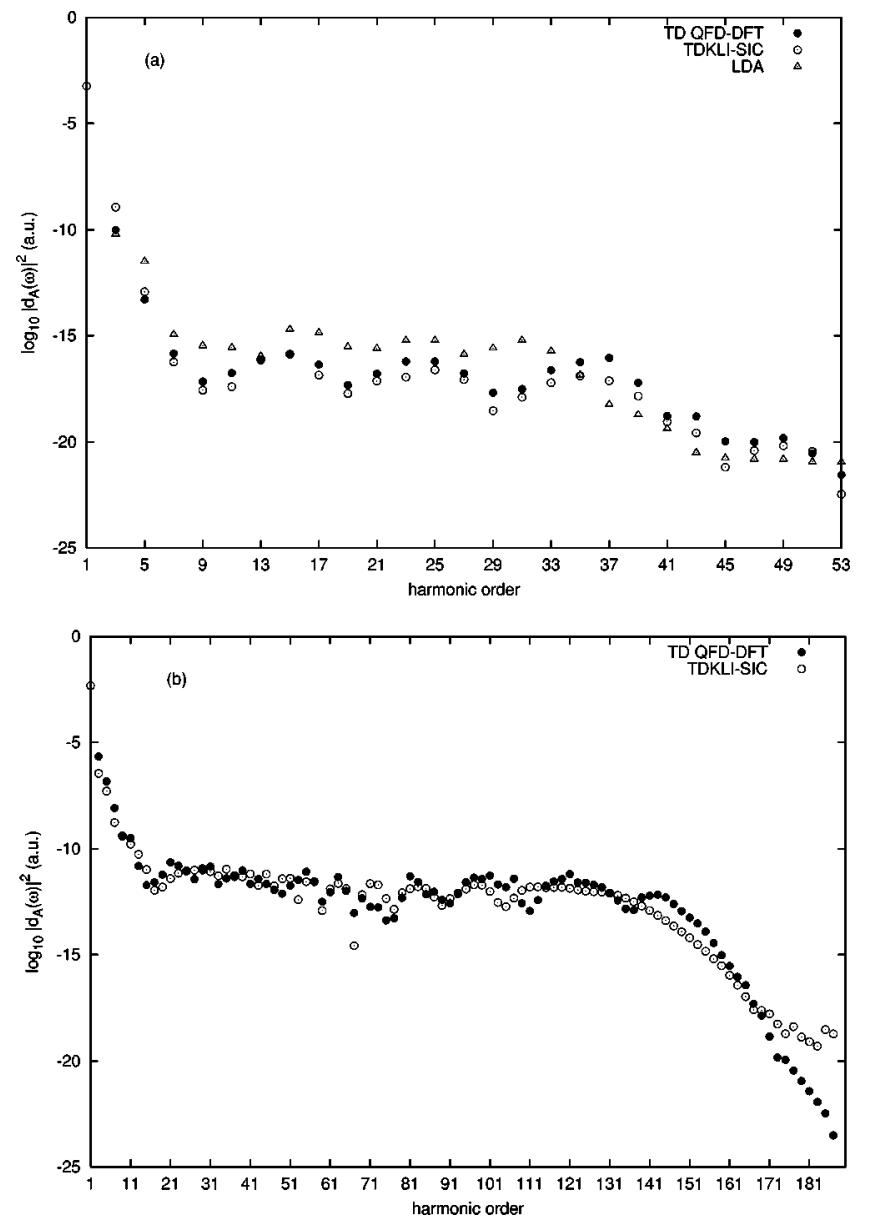

FIG. 8. Comparison of the HHG spectra of Ne atom. (a) and (b) correspond to the laser intensities of $5 \times 10^{13} \mathrm{~W} / \mathrm{cm}^{2}$ and 4 $\times 10^{14} \mathrm{~W} / \mathrm{cm}^{2}$, respectively. The laser wavelength, pulse shape, and pulse lengths are the same as those in Fig. 7.

$2 p$ ) norm obtained from the more accurate all-electron TD KLI-SIC calculations [4]. The overall agreement is satisfactory, well within $0.1 \%$. Figure 7 (a) additionally presents the result from the TD QFD-DFT calculation but using the LDA$X$ potential. In the latter case, the norm $N(t)$ falls off more rapidly than other more accurate calculations. Similar to the He case, this can be attributed to the weaker binding of the LDA electrons arising from the lack of correct asymptotic Coulombic-potential tail. Note that the present approach does not distinguish the individual orbital components and, therefore, the $N(t)$ in the present calculation does not directly correspond to the TD KLI-SIC results. However, since the ionization probability is considerably higher for the $2 p$ and $2 s$ orbitals compared with the $1 \mathrm{~s}$ orbital, it still gives a quantitative comparison.

Figures 8(a) and 8(b) show the comparison of the calculated HHG power spectra for $\mathrm{Ne}$ obtained by (i) the present TD QFD-DFT approach using the XC potentials given by Eqs. (2.16) and (2.17), and (ii) the all-electron TD KLI-SIC approach [4], for the two laser intensities $5 \times 10^{13}$ and 4 $\times 10^{14} \mathrm{~W} / \mathrm{cm}^{2}$, respectively. All other laser parameters are the same as those in Fig. 7. Figure 8(a) also shows the result for the TD QFD-DFT calculation using the LDA- $X$ potential, and the effect is similar to that noticed in $\mathrm{He}$, namely, the LDA electrons tend to have higher HHG plateau intensity but a shorter cutoff. The overall agreement of the present TD QFD-DFT results (with full XC potentials) with the allelectron TD KLI-SIC data [4] are considerably closer, though not perfect. It is gratifying that the present singleorbital TD QFD-DFT approach can provide such a semiquantitative accuracy, suggesting that the procedure may be extendable to considerably larger systems where the ab initio TD KLI-SIC method becomes inaccessible. The main source of error in the present QFD calculations may be attributed to the approximation used in the $T_{\text {corr }}$ term in the kinetic-energy functional.

\section{CONCLUSION}

We have investigated the feasibility and usefulness of extending the QFD-DFT approach for quantitative study of many-electron systems in strong fields. Instead of solving $N$ coupled nonlinear partial-differential equations (PDEs) (as in the TDHF or TDDFT approaches), the present methodology requires one to solve only one single nonlinear Schrödingerlike PDE in terms of the 3D single-particle density for the field-driven $N$-electron quantum systems. All other timedependent dynamical properties of interest such as MPI probability and $\mathrm{HHG}$ can be obtained from the timedependent density. The nonlinear hydrodynamical equation in spherical coordinates can be solved accurately and efficiently by the time-dependent generalized pseudospectral method. The procedure is applied to the study of MPI/HHG processes of $\mathrm{He}$ and $\mathrm{Ne}$ atoms in intense laser fields. The results are generally in consonance with those obtained from other recent self-interaction-free TDDFT [4], allowing additional validation of the time-dependent QFD-DFT approach. We also explore the role of exchange and correlation on the MPI/HHG processes of He in details by using four different $\mathrm{XC}$ energy functionals.

The present methodology can be extended, in principle, to the study of other more complex atomic and molecular systems in strong fields. The success of the method will be, however, dictated by the choice and availability of suitable kinetic and XC energy functionals. Approximate but reliable kinetic energy functionals for larger noble-gas atoms (Ne, Ar, $\mathrm{Kr}$, and $\mathrm{Xe}$ ) are already available [35]. Development of universal and more accurate kinetic energy functionals in QFDDFT formalism will be highly desirable. The unique feature of the QFD-DFT single-equation approach lies in its simplicity and it can provide a powerful and practical method for large systems, where other TDDFT approaches become computationally infeasible. More work on this direction is in progress.

\section{ACKNOWLEDGMENTS}

This work was partially supported by U. S. Department of Energy, Office of Science, Office of Basic Energy Sciences, Division of Chemical Sciences. We acknowledge the Kansas Center for Advanced Scientific Computing for providing the Origin2400 supercomputer time. 
[1] Z. Chang, A. Rundquist, H. Wang, M.M. Murnane, and H.C. Kapteyn, Phys. Rev. Lett. 79, 2967 (1997).

[2] C. Spielmann, N.H. Burnett, S. Sartania, R. Koppitsch, M. Schnürer, C. Kan, M. Lenzner, P. Wobrauschek, and F. Krausz, Science 278, 661 (1997).

[3] M. Schnürer, C. Spielmann, P. Wobrauschek, C. Streli, N.H. Burnett, C. Kan, K. Ferencz, R. Koppitsch, Z. Cheng, T. Brabec, and F. Krausz, Phys. Rev. Lett. 80, 3236 (1998).

[4] X.M. Tong and S.I. Chu, Phys. Rev. A 57, 452 (1998); 64, 013417 (2001).

[5] X. Chu and S.I. Chu, Phys. Rev. A 63, 023411 (2001).

[6] C.A. Ullrich, U.J. Gossmann, and E.K.U. Gross, Phys. Rev. Lett. 74, 872 (1995).

[7] See, for example, R.G. Parr and W. Yang, Density-Functional Theory of Atoms and Molecules (Oxford University Press, New York, 1989).

[8] J.B. Krieger, Y. Li, and G.J. Iafrate, Phys. Lett. A 146, 256 (1990).

[9] X. Chu and S.I. Chu, Phys. Rev. A 64, 063404 (2001).

[10] E. Madelung, Z. Phys. 40, 332 (1926).

[11] L. de Broglie, Compt. Rend. 183, 447 (1926).

[12] D. Bohm, Phys. Rev. 85, 166 (1952).

[13] B.K. Dey, A. Askar, and H.A. Rabitz, J. Chem. Phys. 109, 8770 (1998).

[14] F.S. Mayor, A. Askar, and H.A. Rabitz, J. Chem. Phys. 111, 2423 (1999).

[15] R.E. Wyatt and E.R. Bittner, J. Chem. Phys. 113, 8898 (2000).

[16] R. Wyatt, D.J. Kouri, and D.K. Hoffman, J. Chem. Phys. 112, 10730 (2000).

[17] F. Bloch, Z. Phys. 81, 263 (1928).

[18] L.H. Thomas, Proc. Cambridge Philos. Soc. 23, 542 (1926).

[19] E. Fermi, Z. Phys. 48, 73 (1928).

[20] B.M. Deb and S.K. Ghosh, J. Chem. Phys. 77, 342 (1982).

[21] L.J. Bartolotti, Phys. Rev. A 24, 1661 (1981).

[22] E. Runge and E.K.U. Gross, Phys. Rev. Lett. 52, 997 (1984)

[23] B.M. Deb and P.K. Chattaraj, Phys. Rev. A 39, 1696 (1989).

[24] B.K. Dey and B.M. Deb, J. Chem. Phys. 110, 6229 (1999).
[25] A.K. Roy, B.K. Dey, and B.M. Deb, Chem. Phys. Lett. 308, 523 (1999).

[26] B.M. Deb and P.K. Chattaraj, Phys. Rev. A 39, 1696 (1989).

[27] B.K. Dey and B.M. Deb, Int. J. Quantum Chem. 56, 707 (1995); 70, 441 (1998).

[28] B.K. Dey and B.M. Deb, Chem. Phys. Lett. 276, 157 (1997).

[29] A.K. Roy and B.M. Deb, in Nonlinear Phenomena in Physical and Biological Sciences, edited by S.K. Malik, M.K. Chandrasekharan, and N. Pradhan (INSA, New Delhi, 2000), pp. 947-987.

[30] A. Banerjee and M.K. Harbola, J. Chem. Phys. 113, 5614 (2000).

[31] X.M. Tong and S.I. Chu, Chem. Phys. 217, 119 (1997).

[32] X.M. Tong and S.I. Chu, Phys. Rev. A 61, 012802R (2000).

[33] S.I. Chu and X.M. Tong, Chem. Phys. Lett. 294, 31 (1998).

[34] X.M. Tong and S.I. Chu, Phys. Rev. A 61, 031401R (2000).

[35] B.M. Deb and S.K. Ghosh, Int. J. Quantum Chem. 23, 1 (1983).

[36] S.K. Ghosh and B.M. Deb, J. Phys. B 27, 381 (1994).

[37] G. Brual and S.M. Rothstein, J. Chem. Phys. 69, 1177 (1978).

[38] A.K. Roy, R. Singh, and B.M. Deb, J. Phys. B 30, 4763 (1997).

[39] A.K. Roy, R. Singh, and B.M. Deb, Int. J. Quantum Chem. 65, 317 (1997).

[40] G. Yao and S.I. Chu, Chem. Phys. Lett. 204, 381 (1993).

[41] J. Wang, S.I. Chu, and C. Laughlin, Phys. Rev. A 50, 3208 (1994).

[42] M.R. Hermann and J.A. Fleck, Jr., Phys. Rev. A 38, 6000 (1988).

[43] T.F. Jiang and S.I. Chu, Phys. Rev. A 46, 7322 (1992).

[44] K. Burnett, V.C. Reed, J. Cooper, and P.L. Knight, Phys. Rev. A 45, 3347 (1992).

[45] A.D. Becke, Phys. Rev. A 38, 3098 (1988).

[46] C. Lee, W. Yang, and R.G. Parr, Phys. Rev. B 37, 785 (1988).

[47] X.M. Tong and S.I. Chu, Phys. Rev. A 55, 3406 (1997).

[48] E.R. Davidson, S.A. Hagstrom, S.J. Chakravorty, V.M. Umar, and C.F. Fischer, Phys. Rev. A 44, 7071 (1991).

[49] S.J. Chakravorty, S.R. Gwaltney, E.R. Davidson, F.A. Parpia, and C.F. Fischer, Phys. Rev. A 47, 3649 (1993). 